\title{
Improved Many Machine Excitation Nonlinear Robust Controller Design
}

$$
\text { Qi Yan }{ }^{1, a} \text {, Ying Xia }{ }^{2, b}
$$

${ }^{1}$ College of Information Science and Technology, Bohai University, Jinzhou, 121013, China

${ }^{2}$ Mechanical processing department Jinzhou mechanical and electrical engineering school, Jinzhou, 121013, China

a32392392@qq.com, 'Inxiaying@163.com

Keywords: power system; nonlinear; excitation control; robust stability; backstepping method

\begin{abstract}
Electric power systems are one of nonlinear, multidimensional, dynamic and large scale systems. Once power systems lose stability, it would result in the consequence is very serious. With excitation control for multi-machine power system nonlinear model in this article, a series of stability control problems are studied by means of the improved backstepping methods, including excitation control. The nonlinear adaptive robust controller which can stabilize the system has been designed. The concise design method and excellent design strategy make the designed corresponding control scheme own extensive adaptability, which effectively improve the maintain system power angle and voltage stability.
\end{abstract}

\section{Introduction}

In real life there is a type of electric power system, including two or more than two which can match on the capacity of the power plant, after on the electrical distance is also a big system can match power lines approach transmission power. How to deal with the connection between each subsystem in the power system is a hot issue. Single machine infinite system of excitation control strategies for multi-machine systems may not be suitable [1]. In this case, the research on stability control problem of multi-motor transmission system is very necessary.

The paper focuses on the study of excitation control of multi-machine power system. The adaptive backstepping method is used to design multi-machine system nonlinear robust controller, and further improvement to the traditional adaptive backstepping method is also made, considering both the transient response, and controller gain in the feedback control law of recursive design process. In order to obtain faster convergence speed, the $\mathrm{K}$ class function is referred in the selection of virtual stabilization function. When time tends to go infinity, and the controller gain also tends to be traditional design of the adaptive backstepping method [2], in this way, the transient response speed would be improved significantly and the controller gain doesn't need to increase too much, The adjustable parameters in the $\mathrm{K}$ class function keeps the balance between the controller gain and transient response.

\section{control objectives}

Assuming generator adopts fast excitation mode, build a mathematical model of generator in the power system is as follows:

$$
\left\{\begin{array}{l}
\dot{\delta}_{i}=\omega_{i}-\omega_{0} \\
\dot{\omega}_{i}=-\frac{D_{i}}{H_{i}}\left(\omega_{i}-\omega_{0}\right)+\frac{\omega_{0}}{H_{i}}\left(P_{m i}-P_{e i}\right) \\
\dot{E}_{q i}^{\prime}=-\frac{1+\left(X_{d i}-X_{d i}^{\prime}\right) B_{i i}}{T_{d 0 i}^{\prime}} E_{q i}^{\prime} \\
+\frac{X_{d i}-X_{d i}^{\prime}}{T_{d 0 i}} \sum_{\substack{j=1 \\
j \neq i}}^{n} Y_{i j} E_{q j}^{\prime} \cos \left(\delta_{i j}-\alpha_{i j}\right)+\frac{1}{T_{d 0 i}} V_{f i}
\end{array}\right.
$$

Among them, the one generator active power: 


$$
P_{e i}=E_{q i}^{\prime 2} G_{i i}+E_{q i}^{\prime} \sum_{\substack{j=1 \\ j \neq i}}^{n} E_{q j}^{\prime} Y_{i j} \sin \left(\delta_{i j}-\alpha_{i j}\right)
$$

Where, $\delta_{\mathrm{i}}$ is the $\mathrm{i}$ electric potential generator system a node voltage vector $\bar{E}$ i and the angle between vectors $\bar{V}_{\text {REF }}$, said first rotor of generator operation angle, rad; $\omega_{\mathrm{i}}$ is the i generator rotor angular speed, rad/s ; $p_{m}$ is the i mechanical power units; $H_{\mathrm{i}}$ is the i moment of inertia units, s; $X_{\mathrm{di}}$ is generator $\mathrm{d}$-axis synchronous reactance, $X_{\mathrm{di}}$ is $\mathrm{d}$-axis transient reactance; $T_{d 0 i}$ is the $\mathrm{i}$ generator excitation winding time constant, $\mathrm{s} ; D_{i}$ is the i damping coefficient generator; $E_{q i}^{\prime}$ is the $\mathrm{i}$ generator q-axis transient electric potential; $E^{\prime}{ }^{\prime} \mathrm{j}$ is the $\mathrm{j}$ generator q-axis transient electric potential; rad; $G_{i i}$ and $Y_{i j}$ is respective the i node conductance and mutual admittance between the $\mathrm{i}$ an ${ }^{\delta_{i i}}=\delta_{i}-\delta_{j} \mathrm{~d}$ the $\mathrm{j}$ node[3]; $\alpha \mathrm{i}_{j}$ is the impedance Angle of complement. ${ }^{V_{i}}$ is the $\mathrm{i}$ generator exciting winding voltage as control variables.

To make it into a suitable form of backstepping, make $x_{1}=\delta_{i}-\delta_{i 0}, \quad x_{2}=\omega_{i}-\omega_{i 0}, \quad x_{3}=E_{q i}^{\prime}-E_{q i 0}^{\prime}$, Among them $\delta_{i 0} 、 \omega_{i 0}$ and $E_{q i 0}^{\prime}$ means corresponding to the initial value of the variable. As the known constant, make ${ }^{k=\frac{\omega_{i 0}}{H_{i}}}, b_{1}=X_{q i}-X_{d i}^{\prime}, \quad b_{2}=X_{d i}-X_{d i}^{\prime}$; If the parameters $D_{i}$ is unknown, $\theta=-\frac{D_{i}}{H_{i}}$ is unknown parameter, The (1) system can be further simplified as follows:

$\dot{x}_{1}=x_{2}$

$\dot{x}_{2}=\theta x_{2}+k\left(P_{m i}-\left(x_{3}+E_{q i 0}^{\prime}\right) I_{q i}-b_{1} I_{d i} I_{q i}\right)$

$\dot{x}_{3}=-\frac{x_{3}+E_{q i 0}^{\prime}+b_{2} I_{d i}}{T_{d 0 i}}+\frac{1}{T_{d 0 i}} V_{f i}$

The control target of this paper is aimed at multiple machine excitation control system, by improving the backstepping method to solve the excitation control of multi-motor system. Nonlinear adaptive controller is designed to adjust the generator power angle and frequency to a domain of the steady-state operating point.

\section{The design of nonlinera controller}

The first step: for system (3), $\mathrm{x} 2$ will be regarded as virtual control, and $\mathrm{K}$ classes function will be elected when you pick up the stabilization function[4]. $x_{2}^{*}=-\left[\phi\left(\left|e_{1}\right|\right)+c_{1}\right] e_{1}, \quad c_{1}>0$ is designed for a

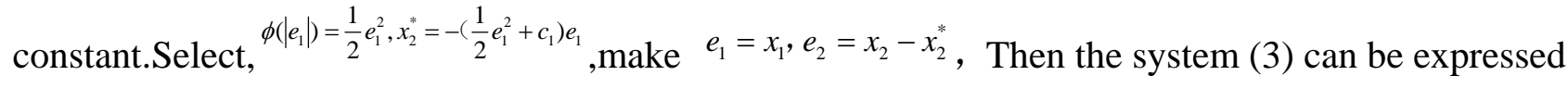
as:

$\dot{e}_{1}=e_{2}-c_{1} X_{1}$

The Lyapunov function for the system (3) can be expressed as:

$\mathrm{v}_{1}=\frac{1}{2} e_{1}^{2}$

It's time derivative $\dot{V}_{1}=e_{1} \dot{e}_{1}=e_{1} x_{2}=e_{1}\left(e_{2}-c_{1} e_{1}-\frac{1}{2} e_{1}^{3}\right)=e_{1} e_{2}-c_{1} e_{1}^{2}-\frac{1}{2} e_{1}^{4}$. It is obvious that when e2=0, $\dot{V}_{1} \leq 0$ [5].

The second step: to augmented (7), forming a new Lyapunov function:

$V_{2}=V_{1}+\frac{1}{2} e_{2}^{2}$

There are: 


$$
\begin{aligned}
& \dot{V}_{2}=\dot{V}_{1}+e_{2} \dot{e}_{2} \\
& =-c_{1} e_{1}^{2}+e_{1} e_{2}+e_{2} \dot{e}_{2}-\frac{1}{2} e_{1}^{4} \\
& =-c_{1} e_{1}^{2}-\frac{1}{2} e_{1}^{4}+e_{2}\left[\begin{array}{l}
e_{1}+\theta x_{2}+k\left(P_{m i}\right. \\
\left.-\left(x_{3}+E_{q i 0}^{\prime}\right) I_{q i}-b_{1} I_{d i} I_{q i}\right)+c_{1} x_{2}+\frac{3}{2} x_{1}^{2} x_{2}
\end{array}\right]
\end{aligned}
$$

Take a new stabilization function:

$$
\begin{aligned}
& x_{3}^{*}=\frac{e_{1}+\theta x_{2}+k\left(P_{m i}-\left(x_{3}+E_{q i 0}^{\prime}\right) I_{q i}-b_{1} I_{d i} I_{q i}\right)+c_{1} x_{2}+\frac{3}{2} x_{1}^{2} x_{2}+c_{2} e_{2}}{k I_{q i}} \\
& =\frac{e_{1}+\theta x_{2}+k P_{m i}+c_{1} x_{2}+\frac{3}{2} x_{1}^{2} x_{2}++c_{2} e_{2}}{k I_{q i}}-E_{q i 0}^{\prime}-b_{1} I_{d i}
\end{aligned}
$$

$c_{2} \geq 0$ is the design of constant. There is $\dot{V}_{2}=-c_{1} e_{1}^{2}-\frac{1}{2} e_{1}^{4}+e_{2}\left(-k I_{q i} e_{3}-c_{2} e_{2}\right)=-c_{1} e_{1}^{2}-\frac{1}{2} e_{1}^{4}-c_{2} e_{2}^{2}-k I_{q i} e_{2} e_{3}$.It is obvious that when $\mathrm{e}_{3}=0, \dot{V}_{2} \leq 0$.

Step 3: define $x=1$, then the formulas (8) augmented, forming a new Lyapunov function can be expressed as:

$V_{3}=V_{2}+\frac{1}{2} e_{3}^{2}$

There is

$$
\begin{aligned}
\dot{e}_{3} & =\dot{x}_{3}-\dot{x}_{3}^{*} \\
& =-\frac{x_{3}+E_{q i 0}^{\prime}+b_{2} I_{d i}}{T_{d 0 i}}+\frac{1}{T_{d 0 i}} V_{f i}-\dot{x}_{3}^{*} \\
& =-\frac{x_{3}+E_{q i 0}^{\prime}+b_{2} I_{d i}}{T_{d 0 i}}+\frac{1}{T_{d 0 i}} V_{f i}-\frac{1}{k I_{q i}}\left[\left(1+3 x_{1} x_{2}+c_{1} c_{2}+\frac{3}{2} c_{2} x_{1}^{2}\right) x_{2}\right. \\
& \left.+\left(\theta+c_{1}+\frac{3}{2} x_{1}^{2}+c_{2}\right)\left(\theta x_{2}+k\left(P_{m i}-\left(x_{3}+E_{q i 0}^{\prime}\right)-b_{1} I_{d i} I_{q i}\right)\right)\right]
\end{aligned}
$$

Then

$$
\begin{aligned}
& \dot{V}_{3}=-c_{1} e_{1}^{2}-\frac{1}{2} e_{1}^{4}-c_{2} e_{2}^{2}-k I_{q i} e_{2} e_{3}+e_{3} \dot{e}_{3} \\
& =-c_{1} e_{1}^{2}-\frac{1}{2} e_{1}^{4}-c_{2} e_{2}^{2}-k I_{q i} e_{2} e_{3}+e_{3}\left(\dot{x}_{3}-\dot{x}_{3}^{*}\right) \\
& =-c_{1} e_{1}^{2}-\frac{1}{2} e_{1}^{4}-c_{2} e_{2}^{2}-k I_{q i} e_{2} e_{3}+e_{3}\left(-\frac{x_{3}+E_{q i 0}^{\prime}+b_{2} I_{d i}}{T_{d 0 i}}+\frac{1}{T_{d 0 i}} V_{f i}-\dot{x}_{3}^{*}\right) \\
& =-c_{1} e_{1}^{2}-\frac{1}{2} e_{1}^{4}-c_{2} e_{2}^{2}-k I_{q i} e_{2} e_{3}+e_{3}\left[-\frac{x_{3}+E_{q i 0}^{\prime}+b_{2} I_{d i}}{T_{d 0 i}}+\frac{1}{T_{d 0 i}} V_{f i}\right. \\
& -\frac{1}{k I_{q i}}\left[\left(1+3 x_{1} x_{2}+c_{1} c_{2}+\frac{3}{2} c_{2} x_{1}^{2}\right) x_{2}+\right. \\
& \left(\theta+c_{1}+\frac{3}{2} x_{1}^{2}+c_{2}\right)\left(\theta x_{2}+k\left(\theta x_{2}+k\left(P_{m i}-\left(x_{3}+E_{q i 0}^{\prime}\right)-b_{1} I_{d i} I_{q i}\right)\right)\right. \\
& \quad V_{3}=-c_{1} e_{1}^{2}-\frac{1}{2} e_{1}^{4}-c_{2} e_{2}^{2}+e_{3}\left\{-k I_{q i} e_{2}-\frac{x_{3}+E_{q i 0}^{\prime}+b_{2} I_{d i}}{T_{d 0 i}}+\frac{1}{T_{d 0 i}} V_{f i}\right. \\
& \quad-\frac{1}{k I_{q i}}\left[\left(1+3 x_{1} x_{2}+c_{1} c_{2}+\frac{3}{2} c_{2} x_{1}^{2}\right) x_{2}+\left(\theta+c_{1}+\frac{3}{2} x_{1}^{2}+c_{2}\right)\left(\theta x_{2}\right.\right. \\
& \left.\left.\quad+k\left(P_{m i}-\left(x_{3}+E_{q i 0}^{\prime}\right)-b_{1} I_{d i} I_{q i}\right)\right)\right]
\end{aligned}
$$

Finally choose feedback control law can be described as follows: 
$V_{f i}=x_{3}+E_{q i 0}^{\prime}+b_{2} I_{d i}+\frac{T_{d 0 i}}{k I_{q i}}\left[\left(1+3 x_{1} x_{2}+c_{1} C_{2}+\frac{3}{2} c_{2} x_{1}^{2}\right) x_{2}\right.$

$+\left(\theta+c_{1}+\frac{3}{2} x_{1}^{2}+c_{2}\right)\left(\theta x_{2}+k\left(\theta x_{2}+k\left(P_{m i}-\left(x_{3}+E_{q i 0}^{\prime}\right)\right.\right.\right.$

$\left.\left.\left.-b_{1} I_{d i} I_{q i}\right)\right)\right]+T_{d 0 i}\left(k I_{q i} e_{2}-c_{3} e_{3}\right)$

$\mathrm{C}_{3}>0$ is considered design constants. Then $\dot{V}_{3}=-c_{1} e_{1}^{2}-\frac{1}{2} e_{1}^{4}-c_{2} e_{2}^{2}-c_{3} e_{3}^{2} \leq 0$.

So assuming that $f\left(x_{1}\right) \neq 0$, The closed-loop error system (11) is asymptotically stable under feedback control law of formula (1)., When $t \rightarrow \infty, \quad e_{1} \rightarrow 0, \quad e_{2} \rightarrow 0, \quad e_{3} \rightarrow 0$.According to the definition of $x_{1} 、 x_{2} 、 x_{3} 、 x_{2}^{*}$ and $x_{3}^{*}, x_{1}$ and $x_{2}$ will converge to zero, $x_{3}$ is also bounded[6].

$\left\{\begin{array}{l}\dot{e}_{1}=e_{2}-c_{1} e_{1}-\frac{1}{2} e_{1}^{3} \\ \dot{e}_{2}=-c_{2} e_{2}-e_{1}-k I_{q i} e_{3} \\ \dot{e}_{3}=-c_{3} e_{3}+k I_{q i} e_{2}\end{array}\right.$

\section{simulation research}

According to the design of the above results, the simulation of closed-loop system on the basis of nonzero initial conditions. Simulation parameters are as

follows: $c_{1}=c_{2}=c_{3}=10, \delta_{0}=\pi / 3, \omega_{0}=314.159, E_{b 1}=50, E_{b 2}=50, E_{b 3}=50, \mathrm{D}=3, \mathrm{H}=8$,

$P_{m 0}=0.9, I_{d}=0.22, I_{q}=1, E_{q 0}=0.4, X_{q}=2.5, X_{d}=2.5, \mathrm{X}_{d}^{\prime}=0.25, T_{d 0}=8$. As shown in figure 1 is responding curves of angle error response curve simulation diagram, shown in figure 2 is responding curves of speed error simulation diagram [7].

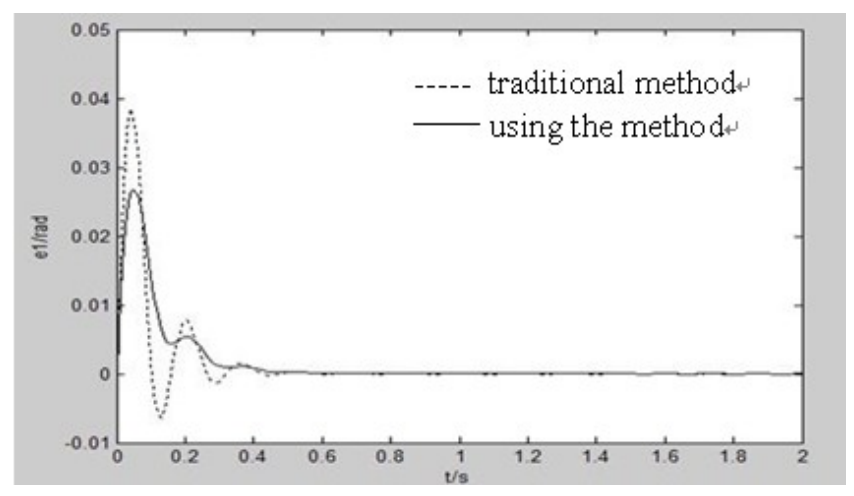

Fig.1. Responding curves of the Power angle error

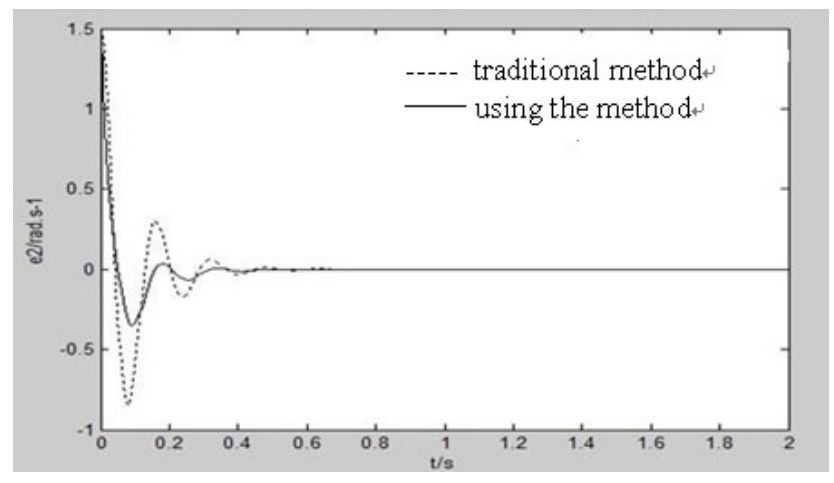

Fig.2. Responding curves of speed error response

From the state of the system response curve a conclusion can be drawn, the system convergence speed is quicken and it moves into a stable state in a very short period of time. Although as you can see from the figure. Although based on traditional adaptive Backstepping method is used traditionally to design the generator excitation controller and also can make the system stabilization[8], but the controller designed with the improved adaptive Backstepping method proposed in this paper, due to the introduction of the $\mathrm{K}$ class function, quickens system convergence speed. 


\section{Conclusion}

For multi-machine excitation system, this paper has introduced the adaptive backstepping method, and the backstepping method is improved. In the recursive design of the feedback control law, when virtual stabilization function is selected, the $\mathrm{K}$ class function is introduced. With the result that when the error is bigger, the convergence rate of the system speeds up[9]. But with the increase of t, the error is reduced. Using improved backstepping method starting from the model description to design the controller, in the end, the particular form of the controller is obtained. Due to the design process without any linearization, the original system and the nonlinear characteristic of the system is retained completely [10]. At the same time, power network parameters is contained in the control law, Which contributes to the decentralized control. And the design process is simple, easy to be accept engineering personnel. At the same time, the simulation analysis shows that this kind of controller can improve transient stability of the system.

\section{Acknowledgment}

This work is supported by social science fund project of Liaoning province (L14BTJ002): Based on analysis of big data research the guidance of college students' learning and future employment

\section{References}

[1] H. Gao, T. Chen, J. Lam. A new delay system approach to network based control. Automatica. 2008, 44: 39-52

[2] S. Yin, S. Ding, A. Haghani, H. Hao, P. Zhang. A comparison study of basic datadriven fault diagnosis and process monitoring methods on the benchmark Tennessee Eastman process. Journal of Process Control, 22(9):1567-1581, 2012.

[3] S. Ding, P. Zhang, S. Yin, E. Ding , An integrated design framework of fault-tolerant wireless networked control systems for industrial automatic control applications, IEEE Transactions on Industrial Informatics, 9(1): 462-471, 2013

[4] Hu Z,Chen Y,Fan D,et al.A novel Power system stabilizer based on neural network inverse system, 2004 International Conference on Power System Teehnology, SingaPore, 2004(l): $75-80$

[5] S. Yin, H. Luo, S. Ding, Real-time implementation of fault-tolerant control systems with performance optimization, IEEE Transactions on Industrial Electronics, 64(5):2402-2411, 2014

[6] Haque M H,Kumkratug P.APPlication of Lyapunov stability criterion to determine the control strategy of a STATCOM, IEE Proeeedings:Generation,Transmission and Distribution, 2004, 151(3): 415-420

[7] X. Li and H. Gao. A Heuristic Approach to Static Output-Feedback Controller Synthesis with Restricted Frequency-Domain Specifications. IEEE Trans. Autom. Control, 2014. DOI:10.1109/TAC.2013.2281472

[8] Chen,H,Ji,H B,Wang B,Xi H S.Coordinated Passivation teehniques for the dual-excited and steam-valving control of synehronous generators,IEE proceedings of Control Theory and Applieations, 2006, 153(1): 69-73

[9] AL-Baiyat S A.Power system transient stability enhancement by STATCOM with nonlinear $\mathrm{H}$ ostabilizer,Eleetrie Power Systems Researeh, 2005, 73(1): 45-52

[10] S. Yin, G. Wang, H. Karimi, Data-driven design of robust fault detection system for wind turbines, Mechatronics, DOI:10.1016/j.mechatronics. 2013.11.009, 2013 